\title{
Analysis of Book Shelf Utilization Rate of University Library Based on DEA Model
}

\author{
Xu Chuanjie \\ Library of Jianghan University \\ Jianghan University, Wuhan, 430056, P. R. China \\ xcjiejie@yeah.net
}

\begin{abstract}
In order to effectively use book shelves in university library, it is necessary to obtain the accurate readers' demands and extract useful information to improve the utilization rate of book shelves in university libraries. In this paper, a DEA model was set up by using the method of efficiency analysis and evaluation of Data Envelopment Analysis, the model optimization under various conditions were discussed by the survey of different background and personnel information statistics and classified. Finally, the necessity of the weights design of DEA model was proved by the calculation. The result shows that the DEA model is able to analyze the use of bookshelves in university libraries well, which provides a scientific basis for bookshelf layout and book placement in library management.
\end{abstract}

Keywords-DEA; University library; Bookshelf position; Efficiency analysis

\section{INTRODUCTION}

To meet the needs of the readers is the basic purpose of the library service work, and the precondition of the bookshelf book arrangement [1]. At present, what the library is facing with are the changing and diversified demands of readers. Readers' demands are the starting point for bookshelves to carry out the arrangement activities, and the key factors that affect the quality of service of library directly. Therefore, accurately and effectively accessing to information demands of readers, and correctly analyze readers' demand information can not only improve the utilization rate of the shelf effectively, but also significantly improve the service of library to meet the readers ' demands [2].

Data Envelopment Analysis (DEA), an effective method to deal with multi-objective decision-making, is a new efficiency evaluation method based on the concept of relative efficiency [3]. It achieves its own decision-making goals as transform inputs into outputs by evaluating the relative effectiveness of Decision Making Units (DMUs) with a certain amount of inputs and outputs [4]. It is not necessary to pre-assign the weight of the index in the process of evaluation from the inputs and the outputs, which reduces the subjectiveness of the results and makes the results highly objective, therefore, book shelf utilization rate of library was analyzed by the DEA method in this paper.

\section{SHELVE UTILIZATION MODELING}

The basic methods to obtain the information of the university library bookshelf utilization rate analysis are as follows: Through extensive investigation, statistics on the demands information of people with different knowledge background statistics, extracting and summarizing the demands of readers, then plan a reasonable bookshelf arrangement order and book discharge order. Bookshelf utilization planning are composed of three parts: reader demands acquisition, reader demands analysis, bookshelf management planning [5].

(1) Reader demands acquisition: Understand the reader's book attributes and preferences by the Internet technology or issue questionnaires.

(2) Readers' requirement analysis: Cluster readers' demands with similar utility, and establish reader's mathematical model which contains reader's preference and book's attributes, describe the arrangement of bookshelf completely by using the cluster analysis method based on the quantified readers 'utility data.

(3) Bookshelf management planning: Convert the reader demands into bookshelf management weights, and evaluate, analysis and plan them by using the DEA model.

The different categories of bookshelf to be appraised are considered as decision-making units. Assume there are $n$ objects to be evaluated in total, and there are m types of inputs (x) and outputs (y) in each bookshelf, define

$$
h_{j}=\frac{\sum_{r=1}^{s} u_{r} y_{r j}}{\sum_{i=1}^{m} v_{i} x_{i j}}(j=1,2, \cdots, n)
$$

Where the bookshelf $\mathrm{j}$ is efficiency evaluation index, vi is the measure (or weight) of the ith type of input; ui is a metric (or weight) for the type of output; $i=1,2, s ; j=1,2$, n. There is a corresponding efficiency evaluation index in each decisionmaking unit (ie. evaluation object). If the relative validity of the decision-making unit is evaluated and the efficiency evaluation index of all the decision-making units is less than or equal to 1 , then the optimal model is constructed as follows: 


$$
\begin{gathered}
\max \frac{\sum_{r=1}^{s} u_{r} y_{r j}}{\sum_{i=1}^{m} v_{i} x_{i j}} \\
s t\left\{\begin{array}{l}
\frac{\sum_{r=1}^{s} u_{r} y_{r j}}{\sum_{i=1}^{m} v_{i} x_{i j}} \leq 1,(j=1,2, \cdots, n) ; \\
V=\left(v_{1}, v_{2}, \cdots, v_{m}\right)^{T} \geq 0 ; \\
U=\left(u_{1}, u_{2}, \cdots, u_{s}\right)^{T} \geq 0 .
\end{array}\right.
\end{gathered}
$$

After the above model is transformed, it become an equivalent linear model:

$$
\begin{aligned}
& \max \mu^{T} Y_{0}=V_{p} \\
& \text { st }\left\{\begin{array}{l}
\omega^{T} x_{j}-\mu^{T} y_{i} \geq 0 ; \\
\omega^{T} x_{0}=1 ; \\
\omega \geq 0, \mu \geq 0
\end{array}\right.
\end{aligned}
$$

The production possible set is obtained according to the Charnes-Cooper transformation and the dual rule:

$$
T=\left\{(X, Y) \mid \sum_{j=1}^{n} X_{j} \lambda_{j} \leq X, \sum_{j=1}^{n} Y_{j} \lambda_{j} \geq Y, \lambda_{j} \geq 0, j=1,2, \cdots, n\right\}
$$

The DMUs model is:

\section{Min $\theta$}

$$
\text { s.T. }\left\{\begin{array}{c}
\sum \lambda_{j} X_{j} \leq \theta X_{0} \\
\sum \lambda_{j} Y_{j} \geq Y_{0} \\
\lambda_{j} \geq 0 \\
j=1,2, \cdots n
\end{array}\right.
$$

In the model, $\theta$ is the relative efficiency of the decision unit, $\lambda \mathrm{j}$ is the proportion of the $\mathrm{j}$-th decision unit in the effective decision making unit mix comparing to the evaluated unit; $s^{*}$ and $\mathrm{s}^{*}+$ are the relaxation variables, which represent the amount of input redundant and insufficient output respectively[6].

\section{MODEL OPTIMIZATION}

The optimal solution $\theta, \lambda \mathrm{j}, \mathrm{s}^{*}-, \mathrm{s}^{*}+$ can be obtained for each decision unit according to the above model. The details are as the following:

(1) When $\theta=1$ and $s *_{-}=0, s *_{+}=0$, which indicates that the jth decision unit is DEA-valid. This indicates that there is neither the waste of resource use due to excess investment nor the loss of efficiency in resource allocation due to insufficient output for the decision-making unit, which suggests that the decision-making unit is the most rational and configuration efficiency is the highest relatively.

(2) When $\theta=1$ with $s^{*} \neq 0$ or $s^{*}+\neq 0$, it means the jth decision unit is weakly DEA-valid, which indicates that the decision-making unit is relatively optimal, but still can be improved.

(3) When $\theta<1$, it implies that the j-th decision-making unit is non-DEA-valid. This shows that the decision unit is not the resource-optimal resource compared with other decision units.

The results may be that multiple shelves are DEA-valid when using the above DEA model to evaluate the shelf utilization at the same time. In other words, the efficiency index is 1 at the same time, and the bookshelf cannot be sorted and distinguished. In order to overcome this deficiency, virtual shelves An+1 are specially introduced as DMUs in the above model constraints to distinguish the differences between the effective planning schemes effectively. The input and output of $A n+1$ are assumed to be $(x i, n+1 ; y r, n+1)$

$$
\left\{\begin{array}{l}
x_{i, n+1}=\underset{1 \leq j \leq n}{\max \left(x_{i j}\right)} \\
y_{r, n+1}=\underset{1 \leq j \leq n}{\min \left(y_{r j}\right)}
\end{array}\right.
$$

This virtual planning scheme is the worst scheme actually, aims to make the original schemes in the evaluation system more effective compared to this virtual scheme, which makes comparing the efficiency differences of various schemes to the next step so that they can be efficiently sorted in purpose.

\section{DATA ANALYSIS}

The data model must have a clear definition and calculation method, and it is easy to obtain reliable data to ensure the accuracy of data analysis. That is to say, the data index can be measured. There are two criteria, the first is the number can be expressed, and second can be described by behavior. As long as there are in line with one, the data can be measured. Indicators that cannot be expressed in quantity or cannot be described by actions are not operable. They cannot be measured and should be abandoned. Otherwise, they can only be subjective conjectures. Therefore, we try to select the quantifiable indexes of objectivity, not on the subjective fuzzy qualitative evaluation. Its analysis process is shown in Fig. 1.

It is necessary to determine the weights of the DEA model in practical. The weight is a relative concept for a particular indicator. It is the relative importance of an indicator. In a certain period of time, the contribution and importance of each weight for the calculation model is different in the value. Scientifically-weighted design can lead to more efficient allocation and utilization of the limited resources available to them. The available bookshelf resource is limited, and the importance of each indicator varies at a given period. It helps to focus more resources on the books that are more important to the reader by designing weights. 


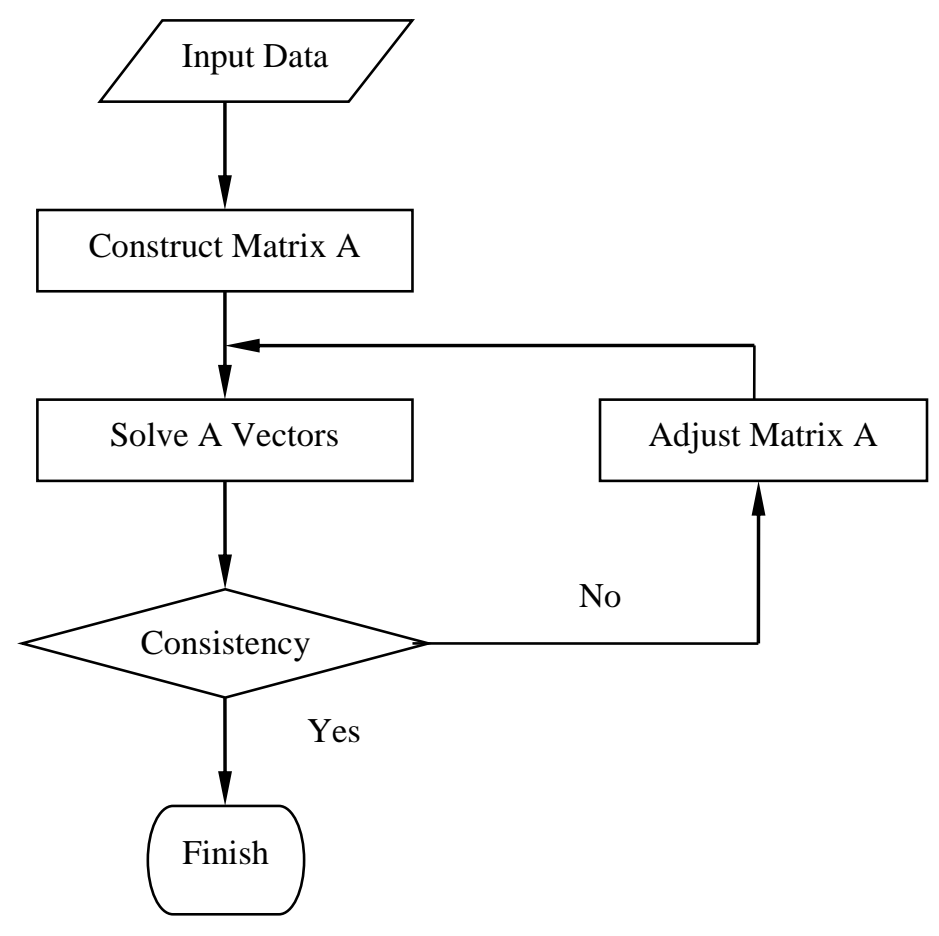

Fig. 1 Calculation process

In this paper, questionnaires were used to investigate the readers' opinions on the relative importance evaluation indicators. The survey questionnaire sent 200 questionnaires in total, and 160 valid questionnaires were received totally. The questionnaire mainly focuses on the reader's needs (A1), the situation of book borrowing (A2) and the condition of bookshelf (A3) in the bookstore, and the book categories (B11), press (B12), the published date (B13), the author (B14), frequency of book borrowing (B21), duration of borrowing (B22), major of borrowers (B23), shelf count (B31), shelves vacancy rate (B32) and so on. The relative importance of each evaluation index can be objectively reflected in general from the view of the composition of the weight, calculate the results of the readers' score in accordance with formula (4) and formula (5), the results are shown in Table I-IV.

TABLE I COMPARING THE MATRIX A1

\begin{tabular}{ccccccc}
\hline A1 & B11 & B12 & B13 & B14 & SCORE & Consistency \\
\hline B11 & 0.24 & 0.03 & 0.21 & 0.33 & 0.21 & 1.01 \\
B12 & 0.31 & 0.24 & 0.48 & 0.29 & 0.42 & 1.79 \\
B13 & 0.22 & 0.28 & 0.22 & 0.38 & 0.34 & 1.23 \\
B14 & 0.45 & 0.35 & 0.03 & 0.12 & 0.15 & 0.35 \\
\hline
\end{tabular}

TABLE II COMPARING MATRIX A2

\begin{tabular}{cccccc}
\hline A2 & B21 & B22 & B23 & SCORE & Consistency \\
\hline B21 & 0.15 & 0.42 & 0.52 & 0.27 & 1.37 \\
B22 & 0.53 & 0.21 & 0.05 & 0.43 & 1.24 \\
B23 & 0.18 & 0.64 & 0.51 & 0.37 & 1.34 \\
\hline
\end{tabular}

TABLE III COMPARING MATRIX A3

\begin{tabular}{|c|c|c|c|c|c|}
\hline A3 & B3 & & B32 & SCORE & Consistency \\
\hline B31 & 0.1 & & 0.09 & 0.18 & 1.90 \\
\hline \multirow[t]{3}{*}{ B32 } & 0.4 & & 0.06 & 0.14 & 1.75 \\
\hline & \multicolumn{2}{|c|}{ TABLE IV } & \multicolumn{3}{|c|}{ COMPARING MATRIX A } \\
\hline & $\mathrm{A} 1$ & $\mathrm{~A} 2$ & A3 & SCORE & Consistency \\
\hline A1 & 0.24 & 0.46 & 0.32 & 0.18 & 1.26 \\
\hline A2 & 0.19 & 0.22 & 0.56 & 0.41 & 1.41 \\
\hline A3 & 0.56 & 0.23 & 0.36 & 0.48 & 1.52 \\
\hline
\end{tabular}

It can be seen that all the indexes calculated above have passed the consistency test and the calculation result is valid.

\section{CONCLUSION}

DEA is a method that compares the efficiency of DMU with multiple inputs and multiple outputs based on linear programming with the unique advantage of objective evaluation results. Although it does not require the relative weight of each evaluation index, it is necessary to distinguish the "input" and "output" variables of each evaluation index, in other words, it requires clearing which ones are input indicators and which ones are output indicators. The university library shelf position analysis based on the DEA method is the multiobjective decision-making method of evaluating the relative validity of the multiindex, which evaluates for purpose of optimization by taking the optimization of utilization rate as the goal and the multiindex input-output weight coefficient as the decision variable. Avoids defining the weight coefficient for purpose of average statistics, equips with the inherent objectivity. The result shows that DEA model established in this paper can analyze the usage of bookshelf in college library well, and provides a scientific basis for setting bookshelf position and books in library management work through analysis. 


\section{REFERENCES}

[1] Fleishhacker J. Collection Development. Stocking Library Shelves for Student Success: Motivating Readers through Science-Focused Fun [J]. Knowledge Quest, 2017, 45.

[2] Johns C W. Rack orientation detection with multiple tags:, WO 2014130368 A1[P]. 2016.

[3] Rahmani M, Ebrahimi B. Determining the Most Efficient Supplier Considering Imprecise Data in Data Envelopment Analysis(DEA), a extension for Toloo and Nalchigar's model [J]. International Journal of Operations \& Production Management, 2017, 13(1).

[4] Rakhshan S A, Kamyad A V, Effati S. Ranking decision-making units by using combination of analytical hierarchical process method and Tchebycheff model in data envelopment analysis[J]. Annals of Operations Research, 2015, 226(1): 505-525.

[5] Suk N. The Effects of Extensive Reading on Reading Comprehension, Reading Rate, and Vocabulary Acquisition [J]. Reading Research Quarterly, 2016, 52 (1).
[6] Mohammadpour M, Hosseinzadeh-Lotfi F, Jahanshahloo G R. An extended slacks-based measure model for data envelopment analysis with negative data $[\mathrm{J}]$. Journal of the Operational Research Society, 2015,66 (7): 1206-1211.

[7] Zhang Yonghong. Library Management Based on 5S Idea [J]. Library and Information Service, 2005, (3): 85 88.

[8] Han J.W., Kamber M. Data Mining: Concepts and Techniques [M]. San Francisco: CA. Morgan Kaufmann, 2011.

[9] Gray Marchionini. Research and development in digital libraries [M]. New York: Marcel Dekker Inc, 1998.

[10] Anwar, M.A. Professional development of librarians working in the university libraries of Pakistan. Library education in Pakistan: Past, present and future. Lahore: Punjab University Library Science Alumni Association. Vol. 9, pp. 65-73, 1991. 